ARTICULOS ORIGINALES Rev Chil Salud Pública 2013; Vol 17 (1): 48-53

\section{Enfermedad de Parkinson y demencia, calidad de vida y sobrecarga del cuidador. Intervención multidisciplinaria en Atención Primaria}

RESUMEN

Introducción: Objetivos del tratamiento de la enfermedad de Parkinson y la demencia son controlar sintomas, conservar funcionalidad y calidad de vida de pacientes y cuidadores y disminuir la sobrecarga del cuidador. Terapia ocupacional y educación tienen utilidad. Objetivo: Evaluar el efecto de una intervención multidisciplinaria en la calidad de vida de cuidadores y pacientes con demencia y/o Parkinson y en la sobrecarga del cuidador. Materiales y método: Diseño cuasiexperimental, 29 pacientes con enfermedad de Parkinson y/o demencia, comuna Vitacura y sus cuidadores. Evaluación de calidad de vida del paciente y cuidador mediante escala WHOQOLBREF y SF-36 respectivamente y la sobrecarga del cuidador mediante escala Zarit. Se realizó intervención con sesiones educativas, talleres de autoayuda y actividades integrativas. Se realizó terapia ocupacional en domicilio. Análisis estadístico con SPSS ${ }^{\circledR}$ versión 17. Resultados: La sobrecarga del cuidador disminuyó significativamente (diferencia promedio de 22 puntos escala Zarit, $p<0,001$ ). No hubo efecto en la calidad de vida de los pacientes, exceptuando una disminución del $22 \%$ de satisfacción en el Dominio "Relaciones Sociales" ( $p 0,001)$. La calidad de vida del cuidador aumentó significativamente en el dominio "Vitalidad" ( $p 0,006)$, con tendencia a mayor satisfacción en el Componente Salud Mental (p 0,077). No hubo diferencias en otros dominios. Discusión: Mediante actividades educacionales y terapia ocupacional, la Atención Primaria en Salud puede ayudar a mejorar la vitalidad, favorecer una mayor satisfacción en la esfera emocional y disminuir la sobrecarga del cuidador, con un efecto más bien neutro en la calidad de vida de pacientes.

Palabras clave: Calidad de Vida, Demencia, Enfermedad de Parkinson, Sobrecarga del cuidador.

\section{Parkinson's disease AND DeMentia, QUALITY OF LIFE, AND CAREGIVER BURDEN: A MULTIDISCIPLINARY PRIMARY HEALTH CARE INTERVENTION}

SEBASTIÁN RosSON ${ }^{1}$, Claudia Fuentealba ${ }^{2}$, Cristian Hormazábal ${ }^{2}$, Carolina Villena ${ }^{2}$ Y Francisca Brieba ${ }^{3}$.

${ }^{1}$ Médico Jefe Programa Visita Domiciliaria CESFAM Vitacura, Indiana 1195 Vitacura, Tel: (02) 7561129 sebastian.rosson@gmail.com ${ }^{2}$ CESFAM Vitacura

${ }^{3}$ Terapeuta Ocupacional, Universidad de Chile.

Financiamiento: Fondos otorgados por el Servicio de Salud Metropolitano Oriente (SSMO) en el marco del Cuarto Concurso de Proyectos de Investigación 2009 del SSMO.

\section{ABSTRACT}

Introduction: Treatment objectives for Parkinson's disease and dementia include controlling symptoms, retaining functionality and quality of life for patients and caregivers, and decreasing caregiver burden. Occupational therapy and education are necessary. Objective: Evaluate the effects of a multidisciplinary intervention on patient and caregiver quality of life for patients with dementia andlor Parkinson's disease, as well as on caregiver burden for those patients. Materials and methods: A quasi-experimental design was applied to 29 patients with Parkinson's disease andlor dementia and their caregivers, in the municipality of Vitacura. Quality of life for patients and caregivers was measured according to the WHOQOLBREF and SF-36 scales; caregiver burden was measured with the Zarit scale. The intervention included educational sessions, self-help workshops, and comprehensive activities. Occupational therapy was offered in patients' homes. SPSS ${ }^{\otimes}$ version 17 
was used for statistical analysis. Results: Caregiver burden decreased substantially (average difference of 22 points on the Zarit scale, $p<0.001$ ). No effect was found in patient quality of life, except for a 22\% decrease in satisfaction in the "Social Relationships" component ( $p 0.001)$. Caregiver quality of life increased significantly in the "Vitality" component ( $p$ 0.006), with a tendency towards greater satisfaction in the "Mental Health" component ( $p$ 0.077). There was no measurable difference in other components. Discussion: Through educational activities and occupational therapy, primary health care can improve vitality, favor a higher level of satisfaction in the emotional sphere, as well as decrease caregiver burden. There is a more neutral effect on patient quality of life.

Key words: Quality of life, dementia, Parkinson's disease, caregiver burden.

\section{INTRODUCCIÓN}

La calidad de vida como constructo multidimensional se define como «la percepción del individuo, de su posición en la vida, en el contexto de la cultura y de los sistemas de valores en los que vive y con relación a sus objetivos, expectativas, normas y preocupaciones» ${ }^{1}$.

La enfermedad de Parkinson y la demencia afectan tanto al individuo como al grupo familiar. Implican trastornos físicos, psicológicos, emocionales, económicos y sociales que deben ser asumidos por el usuario y su familia. Requieren la constante adaptación familiar y conllevan mayor riesgo de aislamiento social, maltrato y desvinculación. La repercusión sobre la atención primaria (APS) se traduce en demanda directa relacionada al paciente o indirecta a través de un cuidador sobrecargado policonsultante.

Estas enfermedades comprometen todas las áreas del funcionamiento y requieren una evaluación multidimensional ${ }^{2,3}$. Existe literatura acerca de la valoración de la calidad de vida en pacientes con Parkinson y estudios que validan la evaluación de calidad de vida en pacientes con demencia leve a moderada ${ }^{4-8}$.

Estas enfermedades son incurables. El objetivo del tratamiento es controlar los síntomas y mantener la mejor funcionalidad posible y conservar la calidad de vida de pacientes y sus cuidadores ${ }^{9,10}$. La utilidad de terapia ocupacional y educación a cuidadores para mejorar la calidad de vida de los pacientes ha sido previamente evaluada con resultados dispares ${ }^{11}$.

\section{MATERIAL Y MÉTODO}

En el universo de pacientes inscritos en el CESFAM Vitacura, se seleccionó una muestra de 29 pacientes con demencia y/o Parkinson y sus cuidadores.
Criterios de inclusión: residente de la comuna, inscrito en el CESFAM, a cargo de un cuidador informal (familiar que no recibe dinero por el cuidado), diagnóstico y/o tratamiento inicial realizado por neurólogo en el nivel secundario de atención de salud.

La clasificación clínica de la severidad de las patologías fue efectuada mediante la escala Clinical Dementia Rating $(\mathrm{CDR})^{12}$ para demencia y escala de Hoen y Yahr ${ }^{13}$ para enfermedad de Parkinson. Para la recolección de información se aplicaron pre y postintervención las siguientes escalas:

1. Escala WHOQOL BREF $F^{14-17}$ heteroaplicada, para medir calidad de vida de los pacientes. Contiene 24 preguntas específicas y dos preguntas globales: calidad de vida global y salud general. Cada ítem tiene 5 opciones de respuesta ordinales tipo Likert. Produce un perfil de cuatro áreas: salud física, psicológica, relaciones sociales y ambiente.

2. Escala $S F-36^{8-20}$, para medir calidad de vida en cuidadores, diseñada por el Health Institute, New England Medical Center, de Boston Massachusetts, que incluye 36 preguntas. Mide ocho conceptos genéricos sobre la salud: Función física, Rol físico, Dolor corporal, Salud general, Vitalidad, Función social, Rol emocional y Salud mental.

3. Escala de Zarit ${ }^{21}$, para medir sobrecarga del cuidador, los puntos de corte son 22 a 46 puntos: sin sobrecarga, 47 a 55 puntos: sobrecarga leve, 56 a 110 puntos: sobrecarga intensa.

4. La autonomía en actividades básicas de la vida diaria (ABVD) se determinó mediante el Índice de Katž ${ }^{22}$.

\section{Descripción de la intervención multidisciplinaria}

Sesiones educativas a cuidadores

- Duración de dos horas cronológicas semanales durante 1 mes, tratándose los siguientes temas: 
a) Fisiopatología, manifestaciones clínicas, diagnóstico, pronóstico de demencia y Parkinson a cargo de médico.

b) Cuidados básicos, aseo y confort de personas dependientes a cargo de enfermera.

c) Alteraciones conductuales y del ánimo en demencia y Parkinson, autocuidado del cuidador, síndrome de sobrecarga del cuidador informal a cargo de psicólogo.

d) Postura, movilización, actividad física y manejo de órtesis a cargo de kinesiólogo.

Sesiones grupales de autoayuda para cuidadores

- Organizadas en 2 grupos guiados por integrantes del equipo, con una duración de 2 horas semanales por un mes. En ellas se trataron temas generativos como la relevancia de su trabajo y situaciones de desgaste.

Actividades integrativas

- Frecuencia semanal por 1 mes con tres horas de duración. Estuvieron a cargo de Enfermera y Terapeuta Ocupacional. Se desarrollaron como actividades competitivas grupales, lúdicas, manuales y físicas con el objetivo de integrar distintos usuarios y cuidadores.

Intervención terapeuta ocupacional en domicilio

- Intervenciones dirigidas a diada paciente-cuidador, una vez a la semana por tres meses, con el objetivo de diagnosticar e intervenir en situaciones de poca participación ocupacional, prevenir el aislamiento social, apoyar en la adaptación del medio ambiente, estructurar rutinas diarias y entrenar la funcionalidad del usuario. Se basaron en intervenciones estandarizadas y fundamentadas en la literatura ${ }^{23,24}$.

\section{Análisis de datos}

Los datos fueron analizados en el programa estadístico SPSS versión 17. Para el análisis descriptivo se utilizaron medias y proporciones. Para la comparación de las medias de satisfacción en las escalas de calidad de vida se uso el $T$ test de student para variables paramétricas depen-dientes.

\section{Aspectos éticos}

El estudio fue aprobado por el Comité de Ética del Hospital del Salvador. La participación en él estuvo fue condicionada a la firma de un consentimiento informado.

\section{RESULTADOS}

La edad promedio de los usuarios fue de 83 años (75 a 96 años). El 60,7\% fueron mujeres, con una proporción de $57,1 \%$ de demencia y $42,9 \%$ de Parkinson. Un 67,9\% de los casos tenía menos de 5 años de evolución.

Los participantes presentaron un grado intermedio de severidad de sus patologías: Escala de Hoen y Yahr promedió 2,7 $\pm 1,2$ y escala CDR promedió $2,1 \pm 0,83$. Respecto al grado de dependencia en las ABVD un $57,7 \%$ de los usuarios presenta Dependencia Leve (Katz A, B o C), mientras 23,1\% tiene Dependencia Severa (Katz F o G).

Respecto a los cuidadores el 65\% fueron mujeres, en un $100 \%$ familiares directos, con una edad promedio de 51 años. El puntaje promedio de la escala de Zarit fue de $54 \pm 15,5$. Un 40\% de los cuidadores con Sobrecarga Intensa y $30 \%$ con Sobrecarga Leve.

Un usuario y su cuidador abandonaron el estudio por traslado a otra comuna con un porcentaje de pérdida de seguimiento de $3,5 \%$.

\section{Intervención}

Entre abril y julio de 2010 se llevaron a cabo sesiones educativas (promedio de asistencia de $72 \%$ ), sesiones de grupo de autoayuda (58\% promedio de asistencia) y sesiones integrativas (asistencia promedio de 51\%). La asistencia global a las actividades presenciales fue de $60 \%$. El motivo de inasistencia más relevante fue no contar con apoyo en el cuidado del paciente mientras duraba la sesión. La cobertura de la terapia ocupacional fue de un $95,5 \%$, con un promedio de 11,5 visitas por usuario.

\section{Comparación pre y postintervención}

Se evidenció una disminución significativa del puntaje promedio de la escala de Zarit con una diferencia de 22 puntos ( $\mathrm{p}<0,001)$. Postintervención un $84 \%$ de los cuidadores estaban sin Sobrecarga, un $4 \%$ con Sobrecarga Leve y $12 \%$ con Sobrecarga Intensa. 
Tabla 1. Puntaje promedio en la escala Zarit

\begin{tabular}{|ccccc|}
\hline Inicial & Postintervención & Diferencias de promedios & Intervalo de confianza & Valor P \\
\hline $53,96 \pm 15,5$ & $32,74 \pm 16,1$ & 21,220 & $16,392-26,048$ & $<0,001$ \\
\hline
\end{tabular}

\section{RESULTADOS EN CALIDAD DE VIDA}

Preintervención los dominios con peor evaluación de satisfacción de los pacientes fueron "Estado Físico" y "Salud Emocional". En general cerca del 79\% de los usuarios se encontraba satisfecho o moderadamente satisfecho con su calidad de vida. Postintervención los dominios peor evaluados fueron "Estado físico" y "Relaciones sociales". No hubo diferencias significativas, excepto en "Relaciones Sociales" con un disminución de un $22 \%$ de satisfacción (p 0,001). Cerca del 66\% de los usuarios se encontraba satisfecho o moderadamente satisfecho con su calidad de vida (ver Tabla 2).

En cuanto a los cuidadores, preintervención los dominios con peor evaluación del SF-36 fue- ron "Vitalidad" y "Rol Emocional". En cuanto a los indicadores agregados "Componente Salud Física" (CSF) y "Componente Salud Mental" (CSM), los resultados de satisfacción promedio fueron $74,3 \% \pm 19,7$ y $67,9 \% \pm 22,6$ respectivamente. Se destaca menor satisfacción en la esfera emocional. Postintervención, el dominio "Vitalidad" tuvo una variación positiva estadísticamente significativa (p 0,006). Entre los indicadores agregados destaca una mejoría no significativa de la satisfacción en el Componente Salud Mental $(\mathrm{CSM})$ con una diferencia de 7,15 puntos ( $\mathrm{p}$ $0,07)$. Puede observarse entonces una tendencia al incremento de la calidad de vida en los componentes de salud mental. Los dominios con peor evaluación fueron "Rol físico" y "Salud general" (ver Tabla 3).

Tabla 2. Porcentaje de Satisfacción de Pacientes en la escala WHOQOL-BREF

\begin{tabular}{|lccccc|}
\hline Dominio & Inicial & Postintervención & Diferencias de medias & IC 95\% & Valor P \\
\hline 1: Salud Física & $43,5 \% \pm 18,8$ & $41 \% \pm 16,6$ & 2,467 & $-1,724-6,657$ & 0,227 \\
2: Salud Psicológica & $45,9 \% \pm 18,8$ & $44,3 \% \pm 16,7$ & 1,600 & $-8,544-11,744$ & 0,740 \\
3: Relaciones Sociales & $54,2 \% \pm 24,6$ & $32,2 \% \pm 15,1$ & 22,077 & $10,881-33,273$ & 0,001 \\
4: Ambiente & $64,7 \% \pm 16,2$ & $60,3 \% \pm 11,7$ & 4,400 & $-4,381-13,181$ & 0,301 \\
\hline
\end{tabular}

Tabla 3. Porcentaje de Satisfacción de cuidadores en la escala SF-36

\begin{tabular}{|lrcccc|}
\hline Dominio & Inicial & Post-Intervención & Diferencias de promedios & IC 95\% & Valor P \\
\hline 1: Función Física & $78,46 \%$ & $69,4 \% \pm 26,5$ & 9,038 & $-0,417-18,494$ & 0,006 \\
2: Rol Físico & $75 \%$ & $61,5 \% \pm 36,2$ & 13,462 & $-4,878-31,801$ & 0,143 \\
3: Rol Emocional & $61,5 \%$ & $67,4 \% \pm 35,5$ & $-5,808$ & $-26,782-15,167$ & 0,574 \\
4: Vitalidad & $62 \%$ & $71,8 \% \pm 20,9$ & $-9,923$ & $-16,757--3,090$ & 0,006 \\
5: Salud Mental & $68 \%$ & $69,1 \% \pm 19,3$ & $-1,077$ & $-7,097-4,943$ & 0,716 \\
6: Función Social & $70,3 \%$ & $67,5 \% \pm 27,5$ & 2,808 & $-8,382-13,998$ & 0,610 \\
7: Dolor Corporal & $72 \%$ & $68,9 \% \pm 20,5$ & 3,038 & $-6,422-12,499$ & 0,514 \\
8: Salud General & $62,7 \%$ & $59,8 \% \pm 19,8$ & 2,923 & $-4,028-9,874$ & 0,395 \\
9: CSF & $72 \%$ & $66,1 \% \pm 21,5$ & 7,154 & $-0,820-15,128$ & 0,077 \\
10: CSM & $65,5 \%$ & $70,6 \% \pm 21,9$ & $-3,346$ & $-11,690-4,997$ & 0,417 \\
\hline
\end{tabular}




\section{DISCUSIÓN}

Mediante actividades promocionales, preventivas, educacionales y la colaboración de un terapeuta ocupacional, APS puede aportar en mejorar la vitalidad, disminuir la sobrecarga y ayudar a mejorar la calidad de vida en el componente de salud mental del cuidador de pacientes con demencia o Parkinson. Esto impacta en la vida familiar y en la demanda sobre el sistema de salud. Estas intervenciones son de bajo costo y pueden ser llevadas a cabo por cualquier equipo de APS.

Similar a los hallazgos de otros estudios, la terapia ocupacional, educación y sesiones integrativas tienen un efecto positivo en el cuidador y neutro en el usuario ${ }^{11}$. En nuestro estudio se evidenció una disminución significativa de satisfacción en el dominio WHOQOL-BREF "Relaciones Sociales". Posiblemente durante las sesiones, el contacto frecuente con personas en similares condiciones, las relaciones con sus cuidadores y las experiencias comunes, hayan impactado en su visión previa acerca de su red social, encontrándola menos satisfactoria.

El mayor impacto se logró en la sobrecarga del cuidador con una reducción relativa del puntaje promedio en cerca de un $40 \%$. Factores influyentes pueden ser mayor conocimiento acerca de las patologías, validación del rol de cuidador, de su carga emocional, la asistencia del terapeuta ocupacional en el manejo ambiental y la integración del paciente en las actividades diarias. El contacto con otros cuidadores y la generación de redes influye positivamente.

Realizar este estudio fue una experiencia enriquecedora para el equipo, incluyendo la mirada integral del terapeuta ocupacional. Se usaron implementos técnicos, creativos y espacios físicos disponibles en APS. La recepción de usuarios y cuidadores fue positiva, desarrollándose iniciativas espontáneas para continuar el contacto entre cuidadores. El CESFAM coordinará a través de la municipalidad espacios físicos para las agrupaciones.

Propuestas para continuar en esta línea son replicar este estudio con mayor tamaño muestral, integrando a otros centros de salud y evaluar los resultados a largo plazo.

\section{Agradecimientos}

A la Municipalidad de Vitacura por su interés y disponibilidad. A la Dra. Pía Valenzuela Cornejo, directora del CESFAM Vitacura, por su apoyo y flexibilidad. A la Dra. Patricia Moya Rivera, subdirectora del CESFAM Vitacura por su orientación y consejo. A Isis Muñoz por su valioso aporte, gran experiencia, entusiasmo y entrega.

\section{REFERENCIAS}

1. The WHOQOL Group. The development of the World Health Organization quality of life assessment instrument (The Whoqol) In: Orley J, Kuyken W, editor. Quality of life assessment: international perspectives. Heidelberg. Springer Verlag. 1994.

2. Wilson RS, Goetz CG. Neurologic illness. In: Spilker. B (ed), Quality of life assessment in clinical trials. New York: Raven Press, 1990, pp. 347-356.

3. Steeman E, de Casterlé BD, Godderis J, Grypdonck M. Living with early-stage dementia: a review of qualitative studies. J Adv Nurs 2006; 54(6): 722-38. Review.

4. Schindler JS, Brown R, Welburn P, Parkes. JD. Measuring the quality of life of patients with Parkinson's Disease. En: Walker SR, Rosser RM (Eds.), Quality of Life assessment: key Issues in the 1990's . Dordrecht: Kluwer Academic Publishers, 1993: 289-300.

5. Thorgrimsen L, Selwood A, Spector A, Royan L, de Madariaga López M, et al. Whose quality of life is it anyway? The validity and reliability of the Quality of Life-Alzheimer's Disease (QoL-AD) scale. Alzheimer Dis Assoc Disord 2003; 17(4): 201-8.

6. Trigg R, Jones RW, Skevington SM. Can people with mild to moderate dementia provide reliable answers about their quality of life? Age and Ageing 2007; 36: 1-7.

7. Struttmann T, Fabro M, Romieu G, de Roquefeuil G, Touchon J, Dandekar T, et al. Quality-of-life assessment in the old using the WHOQOL-100: Differences between patients with senile dementia and patients with cancer. Int Psychogeriatr 1999; 11(3): 273-279.

8. Lucas R. Calidad de vida y demencia. Medicina clínica, ISSN 0025-7753, Vol. 128, №. 2, 2007, págs. 70-75. 
9. Redondo A. Terapia ocupacional en Alzheimer y otras demencias. Madrid: Afal; 2005.

10. Hopkins L; Smith D. Terapia Ocupacional. Buenos Aires: Panamericana; 1998.

11. Pulsford D. (1997) Journal of Advanced Nursing 26, 704-709 Therapeutic activities for people with dementia - what, why... and why not?

12. Hughes CP, Berg L, Danziger WL, Coben LA, Martin RL. A new clinical scale for the staging of dementia.Br J Psychiatry. 1982 Jun; 140: 566-72.

13. Hoen MM, Yahr MD., Parkinsonism: onset, progression and mortality. Neurology 1967; 17: 427-442.

14. WHOQOL-BREF Introduction, administration, scoring and version of the assessment. World Health Organization, Geneva 1996.

15. Orley J. Introducción. En: Lucas editor. Versión Española del WHOQOL. Editorial Ergón, Madrid. 1998: 1-3.

16. WHOQOL Group (1998): The World Health Organization WHOQOL-BREF Quality of life Assessment, Psychological Medicine, 28, 551-558.

17. Torres M, Quezada M, Rioseco R, Ducci ME. Calidad de vida de adultos mayores pobres de viviendas básicas: Estudio comparativo mediante uso de WHOQoL-
BREF Rev Méd Chile 2008; 136: 325-333.

18. Encuesta de salud SF-36, Health Institute, NewEngland Medical Center, Boston Massachusetts. Medical Outcome Trust.

19. Ware JE, Snow KK, Kosinski M, Gandek B. SF36 Health Survey Manual and Interpretation Guide. Boston, MA: The Health Institute, New England Medical Center; 1993.

20. Estado de Salud de Beneficiarios del Sistema de Salud de Chile: 2004-2005 Departamento de Estudios y Desarrollo enero 2006.

21. Zarit SH, Reever KE, Bach-Peterson J. Relatives of the impaired elderly: correlates of feelings of burden. Gerontologist 1980; 20: 649-55.

22. Katz $S$ Ford $A B$ et al. Studies of illness in the aged. JAMA 1963; 185: 914-919.

23. Francés I, Barandiaran M, Marcellán T, Intxaurrondo B, Guerrero R. Terapias no farmacológicas en la enfermedad de Alzheimer. Memoria presentada en el Departamento de Salud del Gobierno de Navarra. Pamplona; 2002.

24. Francés I, Barandiarán M, Marcellán T, y Moreno L. Estimulación psicocognoscitiva en las demencias. Anales del Sistema Sanitario de Navarro; 2004; 26 (3): 405-422. 